\title{
Polyphenol-rich dark chocolate: effect on fasting capillary glucose, total cholesterol, blood pressure and glucocorticoids in healthy overweight and obese subjects
}

\author{
S. Almoosawi, E. A. S. Al-Dujaili and L. Fyfe \\ Department of Dietetics, Nutrition and Biological Sciences, Queen Margaret University, Queen Margaret Drive, \\ Musselburgh EH21 6UU, UK
}

Excess cortisol is associated with various variables of the metabolic syndrome including hypertension, insulin resistance and dyslipidaemia $^{(1)}$. This relationship is likely to be mediated via cortisol's ability to regulate NO bioavailability ${ }^{(2,3)}$. Since increased NO bioavailability is considered the main mechanism by which polyphenols improve glucose, blood pressure and lipid homeostasis, the present study aimed to investigate the effect of two doses of polyphenol-rich dark chocolate on fasting capillary glucose (FG), total cholesterol (TC), systolic (SBP) and diastolic blood pressure (DBP) and urinary free cortisol (F), cortisone (E) and cortisone: cortisol (E:F) in a group of overweight and obese subjects.

The study used a single-blind randomised cross-over design wherein fourteen subjects (eight males, six females; age 36 (sD 11) years; BMI 28 (SD 2.5) kg/m²) consumed $20 \mathrm{~g}$ dark chocolate containing $500 \mathrm{mg}$ or $1000 \mathrm{mg}$ polyphenols for 2 weeks, separated by a 1 -week washout period. This $20 \mathrm{~g}$ portion corresponds to half a portion of habitual chocolate intake and provides similar concentrations of polyphenols as used in previous studies on healthy and hypertensive volunteers that have reported significant reductions in blood pressure following consumption of dark chocolate ${ }^{(4,5)}$.

Capillary FG and TC levels were measured using a calibrated Accutrend GC system (Roche Diagnostics, Burgess Hill, West Sussex, UK). Blood pressure was measured using an automated A\&D Medical UA-767 BP monitor (A\&D Medical, San Jose, CA, USA). Subjects also completed three $3 \mathrm{~d}$ diet and physical activity diaries at baseline and during each intervention. Compliance was measured by direct interviewing, returning of empty chocolate foils and assessment of diet diaries.

Repeated-measures ANOVA revealed a significant reduction in capillary FG levels, SBP and DBP following both treatments. No significant differences were observed between dark chocolate containing $500 \mathrm{mg}$ and $1000 \mathrm{mg}$ polyphenols, suggesting that both doses were equally effective in improving these variables $(P>0.05)$. No changes in anthropometrical measurements, capillary TC and urinary free glucocorticoids levels were observed.

\begin{tabular}{|c|c|c|c|c|c|c|c|c|c|c|c|c|}
\hline & \multicolumn{6}{|c|}{$1000 \mathrm{mg}$ polyphenols } & \multicolumn{6}{|c|}{$500 \mathrm{mg}$ polyphenols } \\
\hline & \multicolumn{2}{|c|}{ Baseline } & \multicolumn{2}{|c|}{ Week 1} & \multicolumn{2}{|c|}{ Week 2} & \multicolumn{2}{|c|}{ Baseline } & \multicolumn{2}{|c|}{ Week 1} & \multicolumn{2}{|c|}{ Week 2} \\
\hline & Mean & SD & Mean & SD & Mean & SD & Mean & SD & Mean & $\overline{\mathrm{SD}}$ & Mean & $\overline{\mathrm{SD}}$ \\
\hline $\mathrm{FG}(\mathrm{mmol} / \mathrm{l})$ & 4.42 & 0.70 & 4.21 & 0.82 & $3.97 *$ & 0.54 & 4.42 & 0.70 & 3.94 & 0.72 & $3.92 *$ & 0.86 \\
\hline $\mathrm{TC}(\mathrm{mmol} / \mathrm{l})$ & 5.02 & 1.01 & - & - & 4.98 & 0.90 & 5.02 & 1.01 & - & _- & 5.03 & 0.77 \\
\hline $\mathrm{SBP}(\mathrm{mmHg})$ & 119 & 10.5 & 114 & 12.0 & $112 * *$ & 9.68 & 119 & 10.5 & 114 & 9.53 & $112 * *$ & 9.51 \\
\hline $\mathrm{DBP}(\mathrm{mmHg})$ & 78.6 & 7.74 & 74.5 & 7.17 & $74.6 * *$ & 7.39 & 78.6 & 7.74 & 74.6 & 4.27 & $73.0 * *$ & 5.06 \\
\hline $\mathrm{F}(\mathrm{nmol} / \mathrm{d})$ & 77.3 & 27.1 & - & - & 71.2 & 38.9 & 86.8 & 44.1 & - & - & 78.6 & 47.3 \\
\hline $\mathrm{E}(\mathrm{nmol} / \mathrm{d})$ & 54.3 & 26.9 & - & - & 45.8 & 17.3 & 59.6 & 32.6 & - & - & 45.8 & 20.3 \\
\hline E:F ratio & 0.71 & 0.32 & - & - & 0.75 & 0.37 & 0.71 & 0.31 & - & - & 0.63 & 0.21 \\
\hline
\end{tabular}

Mean values were significantly different from those at baseline: $* P<0.05, * * P<0.01$.

In conclusion, the present study confirms previous findings of a reduction in blood pressure and fasting glucose following consumption of polyphenol-rich dark chocolate. This effect seems unlikely to be mediated through the glucocorticoid pathway, although involvement of the renin-angiotensin-aldosterone system cannot be excluded. Furthermore, it appears that increasing the polyphenol dose does not result in further improvement in the assessed variables, suggesting that a saturation effect may occur with increasing doses.

1. Rosmond R \& Björntorp P (2001) Endocrinologist 11, 491-497.

2. Iuchi T, Akaike M, Mitsui T et al. (2003) Circ Res 92, 81-87.

3. Liu Y, Mladinov D, Pietrusz JL et al. (2009) Cardiovasc Res 81, 140-147.

4. Grassi D, Lippi C, Necozione S, Desideri G \& Ferri C (2005) Am J Clin Nutr 81, 611-614

5. Grassi D, Necozione S, Lippi C, Croce G, Valeri L, Pasqualetti P, Desideri G, Blumberg JB \& Ferri C (2005) Hypertension 46, $398-405$. 